\title{
pufC Gene Targeted PCR Primers for Identification and Classification of Marine Photosynthetic Bacterium Rhodovulum sulfidophilum
}

\section{Aoi Koga, Nao Yamauchi, Mayu Imamura, Mina Urata, Tomomi Kurayama, Ranko Iwai, Shuhei Hayashi, Shinjiro Yamamoto and Hitoshi Miyasaka*}

Department of Applied Life Science, Sojo University, Nishiku, Japan

*Corresponding Author: Hitoshi Miyasaka, Department of Applied Life Science, Sojo University, Nishiku, Japan.

DOI: $10.31080 /$ ASMI.2020.04.0770
Received: December 30, 2020

Published: January 28, 2021

(C) All rights are reserved by Hitoshi

Miyasaka., et al.

\begin{abstract}
The marine non-sulfur purple photosynthetic bacterium Rhodovulum sulfidophilum has a wide application potential in the fields of aquaculture, renewable energy production, environmental protection, and biomaterial production. To detect, identify and classify various $R$. sulfidophilum strains, we designed a PCR primer set targeting pufC gene encoding one of the photosystem proteins. Nucleotide sequence alignment of the pufC genes from five $R$. sulfidophilum strains revealed that the 3 ' region of this gene is rich in nucleotide substitutions (approximately 10 substitutions/100 bp), making it suitable for the identification and classification of various $R$. sulfidophilum strains. We designed a primer set that amplified $0.7 \mathrm{~kb}$ of the 3 ' region of pufC gene. For the validation of this primer set, we used fish fecal DNA as the PCR templates, and successfully identified and classified several R. sulfidophilum strains.
\end{abstract}

Keywords: Photosynthetic Bacteria; Rhodovulum sulfidophilum; PCR; Fish Fecal DNA

\section{Introduction}

The marine non-sulfur purple photosynthetic bacterium Rhodovulum sulfidophilum has a wide variety of application potential, such as in probiotics for marine aquaculture [1,2], bioremediation of mercury [3], wastewater treatment [4], nitrogenase-mediated hydrogen production [5,6], and production of biomaterials, including biodegradable plastics [7], RNA-drug [8] and spider silk [9], and biosensors [10]. The PCR-based method for the detection and classification of various $R$. sulfidophilum strains may be a useful tool in many fields of applications. In this study, we designed PCR primers targeting pufC that encodes one of the photosynthetic cytochrome proteins to detect and classify various $R$. sulfidophilum strains from environmental samples.

To validate this primer set, we used fecal DNA from some fish and shrimps as the PCR templates, and successfully detected and classified several $R$. sulfidophilum strains from the intestinal tracts of some fish and kuruma shrimps (Marsupenaeus japonicus).

\section{Materials and Methods}

\section{Bacterial strains}

For the nucleotide sequence alignment of the pufM-pufC region of $R$. sulfidophilum, sequence data from our three original isolates, and 2 sequence data of $R$. sulfidophilum strains MB263 (accession number CP020384) and DSM 1374 (accession number CP015418) from the public database were used.

Our original isolates, were $R$. sulfidophilum OKHT16, KKMI01, and KHHN01 strains. R. sulfidophilum OKHT16 was isolated from Osaka Bay, Japan as described previously [11], and R. sulfidophilum KKMI01 and KHHN01 were isolated from the seashore area 
of Amakusa, Kumamoto, Japan. The GenBank/EMBL/DDBJ accession numbers for the $16 \mathrm{~S}$ rRNA gene sequences of $R$. sulfidophilum OKHT16, KKMI01 and KHHN01 are LC037397, LC596063 and LC596064, respectively.

PCR amplification of the pufM-pufC region of $R$. sulfidophilum strains

PCR amplification of the pufM-pufC regions of $R$. sulfidophilum strains was performed by using genomic DNA as the templates. The PCR primers used were pufM588F (5'- TACTACAACCCGTTCCACGC $-3^{\prime}$ ) and pufC1031R (5'- CATGTCATGGCCGTTCAG -3'). The structure of the pufM-pufC region and the location of the primers are shown in Fig. 1. KOD-Plus DNA Polymerase (Toyobo Life Science, Osaka, Japan) was used for PCR, and the reaction was carried out on a Biometra TOne 96 thermal cycler (Analytik Jena GmbH, Jena, Germany) under the following conditions: pre-denaturation at 94 ${ }^{\circ} \mathrm{C}(2 \mathrm{~min})$, denaturation at $94^{\circ} \mathrm{C}(30 \mathrm{~s})$, annealing at $62{ }^{\circ} \mathrm{C}(30 \mathrm{~s})$, and extension at $68^{\circ} \mathrm{C} \mathrm{(40} \mathrm{s)} \mathrm{for} 30$ cycles, followed by one cycle of final extension at $68{ }^{\circ} \mathrm{C}(10 \mathrm{~min})$. PCR products were separated by $1 \%$ agarose gel electrophoresis, and the pufM-pufC gene fragments were purified. DNA sequence analyses were performed using a commercial DNA sequence analysis service (Eurofins Genomics Inc., Tokyo, Japan). The GenBank/EMBL/DDBJ accession numbers for the pufM-pufC gene fragments of R. sulfidophilum OKHT16, KKMI01 and KHHN01 are LC596067, LC596065 and LC596066, respectively.

\section{Shrimp and fish samples}

Live kuruma shrimps (M. japonicus) for laboratory experiments were provided by the shrimp pond of Takusui Co. Ltd. located in Imari, Saga, Japan. Wild-caught kuruma shrimps from Oita Bay and Seto Inland Sea, Japan, were purchased by online. Wild-caught ocean fish such as round herring (Etrumeus teres), konoshiro gizzard shad (Konosirus punctatus), and righteye flounder (Pseudopleuronectes herzensteini) and freshwater fish such as carp (Cyprinus carpio), iwana (Salvelinus leucomaenis) and loach (Misgurnus anguillicaudatus) were purchased from local markets or online.

Isolation of fecal DNA from shrimps and fish, and PCR amplification of the 3' region of pufC

Isolation of fecal DNA was performed using the ISOFECAL kit (Nippon Gene, Tokyo, Japan). PCR amplification, DNA purification by agarose gel electrophoresis, and DNA sequencing were performed as described in section 2.2.

The GenBank/EMBL/DDBJ accession numbers for the pufC fragments obtained by PCR amplification from fecal DNA are shown in the figure legend of figure 3 .

\section{Phylogenetic analysis}

Phylogenetic analysis was performed on the Phylogeny.fr platform (http://www.phylogeny.fr) [12] using its default setting. Branch support in the maximum likelihood (ML) tree was estimated with 100 bootstrap replicates.

\section{Results}

PCR primer design for the amplification of a part of pufC gene of $R$. sulfidophilum

To detect, identify and classify $R$. sulfidophilum, we designed a PCR primer set for the amplification of a part of pufC encoding one of the cytochrome proteins involved in electron transfer to the photosynthetic reaction center [13]. For the identification of PSB from non-purified cultures, the pufL-pufM region of the puf cluster has been used $[14,15]$, because nucleotide sequences of pufL-pufM are well conserved among various PSB. In this study, to detect and identify $R$. sulfidophilum strains, pufC gene was selected as the target, because of its unique location in the puf cluster in R. sulfidophilum compared to other genera of photosynthetic bacteria, and the absence of this gene in several closely related photosynthetic bacteria [13].

First, to obtain the nucleotide sequence data of $p u f M-p u f C$ from our three original isolates, we designed a primer set. To design the forward primer in the pufM region, the nucleotide sequences of pufM genes from seven Rhodovulum sp., namely R. sulfidophilum (AB020784), R. sulfidophilum DSM 2351 (AP014800), R. kholense type strain JA297T (FM208076), $R$. visakhapatnamense type strain JA181T (AM944097), R. sp. MTCH3IM048 (FN984739), R. euryhalinum (AF486825), and R. marinum type strain JA128T (AM944096) were aligned using CLUSTAL W (1.83) multiple sequence alignment program. The forward primer pufM588F (5' - TACTACAACCCGTTCCACGC - 3 ') was designed in the well-conserved region in the alignment. To design the reverse primer in the pufC region, the nucleotide sequences of three Rhodovulum $s p$., namely $R$. sulfidophilum (AB020784), R. sulfidophilum DSM 2351 (AP014800), Rhodovulum 
sp. OG-KC1M (AB088691) were aligned using CLUSTAL W (2.1) multiple sequence alignment program. Based on these alignments, we found several conserved regions in $p u f C$ gene, and designed the reverse primer pufC1031R (5'- CATGTCATGGCCGTTCAG -3'). The alignment data for the design of pufM588F and pufC1031R primers will be provided on request.

The nucleotide sequences of pufM-pufC from the three original R. sulfidophilum isolates (strains OKHT16, KKMI01 and KHHN01), were determined by PCR amplification of the genes with pufM588F and pufC1031R primers.

Next we aligned the pufC sequences of $R$. sulfidophilum strains OKHT16, KKMI01 and KHHN01, and two R. sulfidophilum strains in the database to design the primers (The alignment data will be provided on request). To attain a higher resolution for the classification of various $R$. sulfidophilum strains, we determined the region of pufC gene with many nucleotide substitutions. Fig. 1 shows the numbers of nucleotide substitution positions (substitution positions/50 bp) found in the alignment of five R. sulfidophilum strains. We found that the 3 ' region of this gene is rich in nucleotide substitutions, and is suitable for the classification of different $R$. sulfidophilum strains. Additionally, we found several well-conserved (less number of substitutions) regions in $p u f C$, and two of these regions were found to contain heme-binding sites (indicated by arrows in Fig. 1). Based on these findings, we designed the forward primer pufC336F (5'-AACTTCGACCAGCTCACCAA -3') in the conserved region as shown in Fig. 1. PCR amplification of genomic DNA of $R$. sulfidophilum with pufC336F and pufC1031R primers generated $690 \mathrm{bp}$ of the 3 ' region of pufC. The targeted region is rich in nucleotide substitutions among $R$. sulfidophilum strains, making it suitable for the identification and classification of various $R$. sulfidophilum strains.

\section{Validation of the primer set}

For the validation of the primer set, we amplified the target region of pufC by using fecal DNA from shrimps and fish as PCR templates, because $R$. sulfidophilum is one of the promising candidates for probiotics in aquaculture [1,2]. Initially, we maintained kuruma shrimps (M. japonicus) from a commercial shrimp pond in laboratory aquaria, and fed them with a diet containing $R$. sulfidophilum as probiotics, and tried to detect $R$. sulfidophilum from their fecal DNA. As a result, however, we unexpectedly detected $R$. sulfidophilum from both $R$. sulfidophilum-fed shrimps and non-fed (control) shrimps (data not shown). This result suggests that $R$. sulfidophilum may commonly inhabit in the intestinal tract of kuruma shrimps. Based on this finding, we next tried to amplify pufC from the fecal DNA of wild-caught kuruma shrimps as well as from some other wild-caught ocean fish. As expected, being a marine PSB, $R$. sulfidophilum did not inhabit in the intestinal tracts of freshwater fish, and the fecal DNA of some freshwater fish was used as a negative control. As for ocean fish, round herring (E. teres), konoshiro gizzard shad (K. punctatus), and righteye flounder (P. herzensteini) were studied, and for freshwater fish, carp (C. carpio), iwana (S. leucomaenis) and loach (M. anguillicaudatus) were examined.

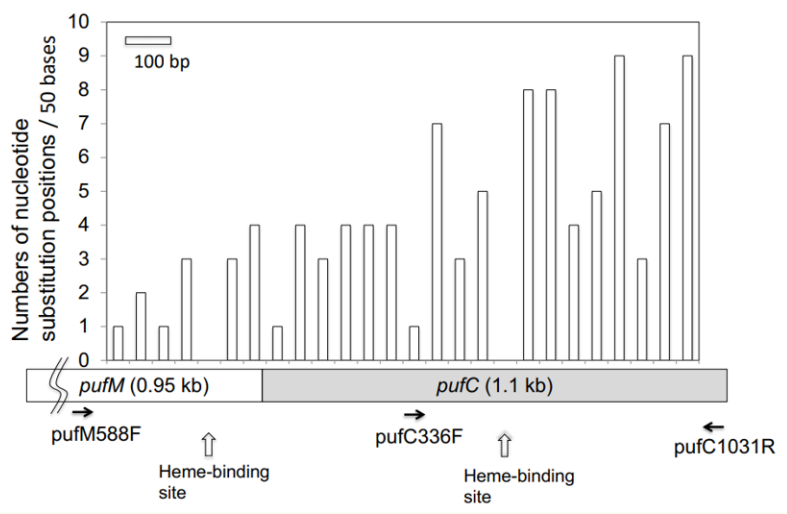

Figure 1: Structure of $p u f M$ - pufC, and numbers of nucleotide substitution positions in pufM - pufC among five Rhodovulum sulfidophilum strains.

The nucleotide sequences of the pufM - pufC region of $R$. sulfidophilum DSM 2351 (AP014800) and DSM 1374 (CP015418) in the database, and those from our original isolates ( $R$. sulfidophilum OKHT16, KKMI01, and KHHN01) were aligned using ClustalW, and the numbers of nucleotide substitution positions per $50 \mathrm{bp}$ were counted. The arrows show the positions of the primers. The heme-binding sites of PufC are indicated by vertical arrows.

Figure 2 shows the results of agarose gel electrophoresis of the PCR products. We successfully amplified pufC genes from all the fecal DNA samples of wild-caught kuruma shrimps and ocean fish. For freshwater fish, pufC was not amplified from carp (C. carpio), 
but was unexpectedly amplified from iwana (S. leucomaenis) and loach (M. anguillicaudatus). In addition, we performed PCR amplification of DNA from two freshwater fish belonging to the Cyprinidae family, barbel steed (Hemibarbus barbus) and Japanese crucian carp (Carassius cuvieri), and pufC was not amplified from the fecal DNA of these fish (data not shown).

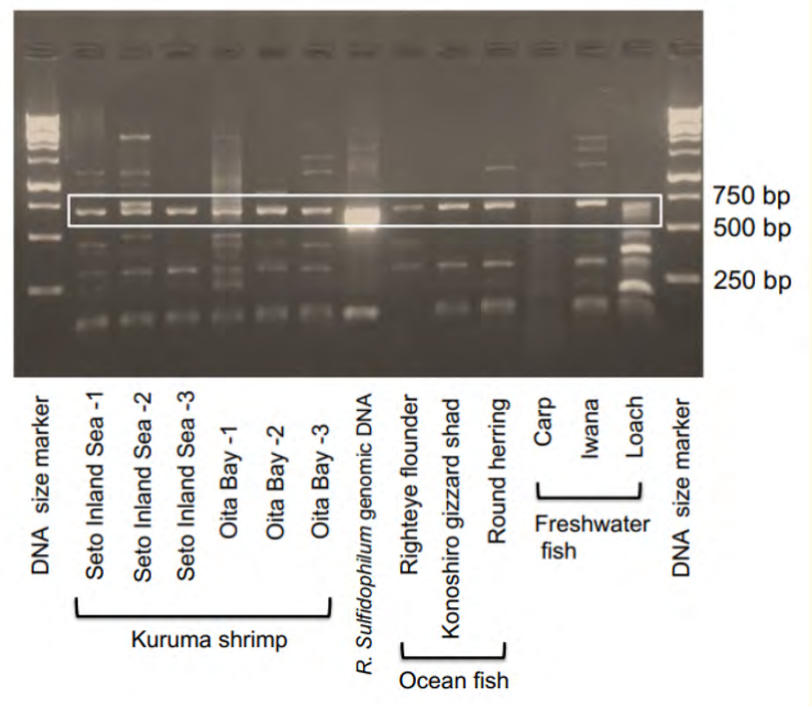

Figure 2: Agarose gel electrophoresis of PCR fragments of pufC gene.

The fecal DNA form six wild-caught kuruma shrimps (three from Seto Inland Sea and three from Oita Bay), three ocean fish (round herring, konoshiro gizzard shad, and righteye flounder), and three freshwater fish (carp, iwana and loach) were amplified using PCR with the primer set of pufC336F and pufC1031R. The 690 bp PCR fragments are indicated by a white box.

Figure 3 shows the phylogenic tree drawn with the nucleotide sequences of PCR-amplified fragments of pufC of five R. sulfidophilum strains. All the PCR-amplified fragments were separated at a good resolution, and most $R$. sulfidophilum strains detected from fish and shrimp fecal DNA were in the same group as R. sulfidophilum KKMI01 and KHHN01 strains. The GenBank/EMBL/DDBJ accession numbers for $p u f C$ fragments amplified from the fecal DNA from fish and shrimps are shown in the figure legend of figure 3.

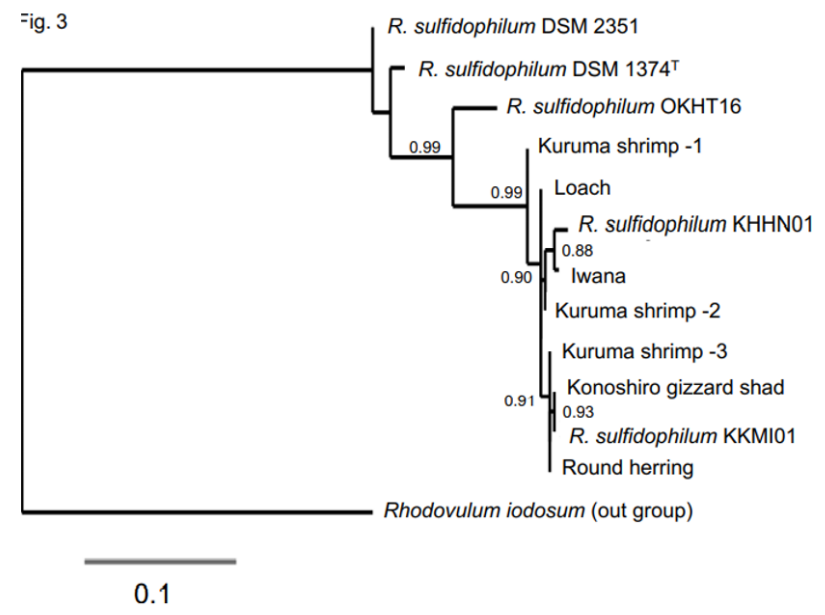

Figure 3: Phylogenetic tree constructed by the maximum likelihood (ML) method based on the nucleotide sequences of PCR fragments of $p u f C$ genes obtained from the fecal DNA of wild-caught fish and shrimps.

The GenBank/EMBL/DDBJ accession numbers for pufC gene fragments are LC596358 (Round herring), LC596359 (Konoshiro), LC596360 (Loach), LC596361 (Shrimp -1), LC596362 (Shrimp -3), LC596363 (Shrimp -2), and LC596364 (Iwana). The GenBank/EMBL/DDBJ accession numbers for pufC genes of $R$. sulfidophilum strains are CP015418 (DSM 1374 type culture), AP014800 (DSM 2351), LC596067 (OKHT16), LC596065 (KKMI01) and LC596066 (KHHN01), and that for Rhodovulum iodosum (out group) is AB088689.

\section{Discussion}

The marine photosynthetic bacterium, R. sulfidophilum, has a high potential for practical applications in various fields. In this study, a PCR primer set to detect, identify and classify various $R$. sulfidophilum strains was designed. The nucleotide sequence alignment of pufC of five $R$. sulfidophilum strains revealed the substitution-rich part in the 3 ' region of this gene, and a primer set targeting this region was designed. This primer set can be used for the identification and classification of $R$. sulfidophilum, from samples of i) pure $R$. sulfidophilum cultures, ii) non-purified cultures of $R$. sulfidophilum, and iii) environmental samples, such as fecal DNA. 
As a validation of this primer set, we successfully amplified $p u f C$ from the fecal DNA of some fish and kuruma shrimps, and classified the nucleotide sequences of PCR fragments, as shown in Fig. 3. In addition, in the process of validation, we obtained some interesting findings. First, our results suggest that $R$. sulfidophilum can commonly inhabit in the intestinal tract of some ocean fish and shrimps. We did not expect this result, but recent metagenomic studies indicated that a relatively large population of PSB was present in the intestinal tract of some aquatic animals $[16,17]$, consistent with our findings. Rhodovulum sulfidophilum belongs to the class Alphaproteobacteria, order Rhodobacterales, and family Rhodobacteraceae. In the intestinal tracts of pacific white shrimps, Litopenaeus vannamei, Rhodobacter (a PSB belonging to Rhodobacteracea family) populates in healthy shrimps, and when the shrimps are affected by white feces syndrome, its population was much decreases [18], suggesting that Rhodobacter has some beneficial effects on the health of shrimps. Yamazaki., et al. [19] reported that the presence of Rhodobacterales in the intestinal tracts was related to the enhancement of the growth of sea cucumber, Apostichopus japonicus, suggesting that the polyhydroxybutyrate (PHB) produced by bacterial cells of Rhodobacterales might have some beneficial effects on growth [20]. PHB is a biopolymer produced by some types of bacteria as a storage compound [21], and the beneficial effects of PHB as feed additives for fish and shrimps have also been well investigated [22,23]. Rhodovulum sulfidophilum is a PHB-producing bacterium [7], and may have some beneficial effects on fish and shrimps. Cristiane., et al. reported that Rhodovulum was a relevant constituent of the sponge-associated microbiome, and its versatile metabolic activities involved in carbon, nitrogen, and sulfate metabolism might have various beneficial effects on host sponges [24]. The results of the present study and previous metagenomic studies suggest that Rhodovulum in the intestinal tracts of aquatic animals has some beneficial effects on the host, and is a good candidate for probiotics in aquaculture. Among the R. sulfidophilum strains shown in figure 3, R. sulfidophilum KHHN01 and KKMI01 are closely related to the $R$. sulfidophilum from the fish and shrimps. Thus these two strains may be promising candidates as probiotics in aquaculture. The probiotic effects of $R$. sulfidophilum KKMI01 on kuruma shrimps are being examined in our laboratory and also in outdoor shrimp ponds.

Another interesting finding was the detection of $R$. sulfidophilum, a marine PSB, from the fecal DNA of two freshwater fish, iwana
(S. leucomaenis) and loach (M. anguillicaudatus), and the absence of $R$. sulfidophilum in Cyprinidae fish, carp (C. carpio), barbel steed (H. barbus) and Japanese crucian carp (C. cuvieri). The results shown in the present study are still at a preliminary stage, and more detailed studies are required to clarify the distribution of $R$. sulfidophilum in the intestinal tracts of freshwater fish.

\section{Conclusion}

The marine non-sulfur purple photosynthetic bacterium Rhodovulum sulfidophilum has a wide potential in biotechnological applications. In this study, we designed a PCR primer set targeting pufC gene encoding one of the photosystem proteins to detect, identify and classify various $R$. sulfidophilum strains. For the validation of this primer set, we used fish fecal DNA as the PCR templates, and successfully identified and classified several $R$. sulfidophilum strains. This primer set can be used for the identification and classification of $R$. sulfidophilum, from samples of i) pure R. sulfidophilum cultures, ii) non-purified cultures of $R$. sulfidophilum, and iii) environmental samples, such as fecal DNA.

\section{Acknowledgements}

We would like to thank Takusui Co. Ltd., Fukuoka, Japan, for providing kuruma shrimps from their shrimp ponds.

We would like to thank Editage (www.editage.com) for English language editing.

\section{Conflict of Interest}

The authors declare that there are no conflicts of interest regarding the publication of this article.

\section{Bibliography}

1. Loo PL., et al. "Rhodovulum sulfidophilum, A Phototrophic Bacterium, Grown in Palm Oil Mill Effluent Improves the Larval Survival of Marble Goby Oxyeleotris Marmorata (Bleeker)". Aquaculture Research 44.3 (2013): 495-507.

2. Chang BV., et al. "Investigation of a Farm-Scale Multitrophic Recirculating Aquaculture System with the Addition of Rhodovulum sulfidophilum for Milkfish (Chanos chanos) Coastal Aquaculture". Sustainability (Switzerland) 11.7 (2019).

3. Mukkata K., et al. "Diversity of Purple Nonsulfur Bacteria in Shrimp Ponds with Varying Mercury Levels". Saudi Journal of Biological Sciences 23.4 (2016): 478-487. 
4. Azad SA., et al. "Rhodovulum sulfidophilum in the Treatment and Utilization of Sardine Processing Wastewater". Letters in Applied Microbiology 38.1 (2004): 13-18.

5. Maeda I., et al. "Maximization of Hydrogen Production Ability in High-Density Suspension of Rhodovulum sulfidophilum Cells Using Intracellular Poly(3-Hydroxybutyrate) as Sole Substrate". Biotechnology and Bioengineering 81.4 (2003): 474481.

6. Cai J and Guangce W. "Hydrogen Production by a Marine Photosynthetic Bacterium, Rhodovulum sulfidophilum P5, Isolated from a Shrimp Pond". International Journal of Hydrogen Energy 37.20 (2012): 15070-15080.

7. Foong CP., et al. "Optimal Iron Concentrations for GrowthAssociated Polyhydroxyalkanoate Biosynthesis in the Marine Photosynthetic Purple Bacterium Rhodovulum sulfidophilum under Photoheterotrophic Condition". PLOS ONE 14.4 (2019): $1-12$.

8. Kikuchi Y and Umekage S. "Extracellular Nucleic Acids of the Marine Bacterium Rhodovulum sulfidophilum and Recombinant RNA Production Technology Using Bacteria”. FEMS Microbiology Letters 365.3 (2018).

9. Foong CP., et al. "A Marine Photosynthetic Microbial Cell Factory as a Platform for Spider Silk Production". Communications Biology 3.1 (2020).

10. Fujimoto H., et al. "Whole-Cell Arsenite Biosensor Using Photosynthetic Bacterium Rhodovulum Sulfidophilum". Applied Microbiology and Biotechnology 73.2 (2006): 332-338.

11. Yamauchi N., et al. "Isolation of A Marine Purple Non-Sulfur Photosynthetic Bacterium With A High Ability of Glycerol Assimilation". International Journal of Plant, Animal and Environmental Sciences 9.4 (2019): 214-221.

12. Dereeper A., et al. "Phylogeny.Fr: Robust Phylogenetic Analysis for the Non-Specialist". Nucleic Acids Research 36 (2008).

13. Masuda S., et al. "A New Cytochrome Subunit Bound to the Photosynthetic Reaction Center in the Purple Bacterium, Rhodovulum sulfidophilum". Journal of Biological Chemistry 274.16 (1999): 10795-10801.
14. Tank M., et al. "Phylogenetic Relationship of Phototrophic Purple Sulfur Bacteria According to PufL and PufM Genes". International Microbiology 12.3 (2009): 175-185.

15. Yutin N., et al. "Novel Primers Reveal Wider Diversity among Marine Aerobic Anoxygenic Phototrophs". Applied and Environmental Microbiology 71.12(2005): 8958-8962.

16. Baldo L., et al. "Phylogeography and Ecological Niche Shape the Cichlid Fish Gut Microbiota in Central American and African Lakes". Frontiers in Microbiology 10 (2019): 1-19.

17. Ye Q., et al. "Effects of Dietary Gelsemium elegans Alkaloids on Intestinal Morphology, Antioxidant Status, Immune Responses and Microbiota of Megalobrama amblycephala". Fish and Shellfish Immunology 94 (2019): 464-78.

18. Huang Z., et al. "Microecological Koch's Postulates Reveal That Intestinal Microbiota Dysbiosis Contributes to Shrimp White Feces Syndrome". Microbiome 8.1 (2020): 1-13.

19. Yamazaki Y., et al. "Repeated Selective Enrichment Process of Sediment Microbiota Occurred in Sea Cucumber Guts". Environmental Microbiology Reports 11.6 (2019): 797-807.

20. Yamazaki Y., et al. "Individual Apostichopus japonicus Fecal Microbiome Reveals a Link with Polyhydroxybutyrate Producers in Host Growth Gaps". Scientific Reports 6 (2016): 1-10.

21. Reddy CSK., et al. "Polyhydroxyalkanoates: An Overview". Bioresource Technology 87.2 (2003): 137-146.

22. Schryver PD., et al. "Poly- $\beta$-Hydroxybutyrate (PHB) Increases Growth Performance and Intestinal Bacterial Range-Weighted Richness in Juvenile European Sea Bass, Dicentrarchus labrax". Applied Microbiology and Biotechnology 86.5 (2010): 15351541.

23. Duan Y., et al. "Effect of Dietary Poly- $\beta$-Hydroxybutyrate (PHB) on Growth Performance, Intestinal Health Status and Body Composition of Pacific White Shrimp Litopenaeus vannamei (Boone, 1931)". Fish and Shellfish Immunology 60 (2017): 520528.

24. Cristiane CP., et al. "Phylogenetically and Spatially Close Marine Sponges Harbour Divergent Bacterial Communities". PLoS ONE 7.12 (2012). 


\section{Assets from publication with us}

- Prompt Acknowledgement after receiving the article

- Thorough Double blinded peer review

- Rapid Publication

- Issue of Publication Certificate

- High visibility of your Published work

Website: www.actascientific.com/ Submit Article: www.actascientific.com/submission.php Email us: editor@actascientific.com

Contact us: +919182824667 